\title{
HOMOLOGICAL DIMENSIONS OF STABLE HOMOTOPY MODULES AND THEIR GEOMETRIC CHARACTERIZATIONS $\left({ }^{1}\right)$
}

BY

\author{
T. Y. LIN
}

\begin{abstract}
Projective dimensions of modules over the stable homotopy ring are shown to be either 0,1 or $\infty$; weak dimensions are shown to be 0 or $\infty$. Also geometric characterizations are obtained for projective dimensions 0,1 and weak dimension 0 . The geometric characterizations are interesting; for projective modules they are about the cohomology of geometric realization; while for flat modules they are about homology. This shows that the algebraic duality between "projective" and "flat" is strongly connected with the topological duality between "cohomology" and "homology". Finally, all the homological numerical invariants of the stable homotopy ring-the so-called finitistic dimensions-are completely computed except the one on injective dimension.
\end{abstract}

1. Introduction and statements of results.

1.1 Introduction. In [7] we investigated the homological dimension of a $\pi_{*}$-module of finitely generated type, where $\pi_{*}$ is the stable homotopy ring. In this paper we extend our computations to general $\pi_{*}$-modules. Namely, we find that the projective dimension of a $\pi_{*}$-module is 0,1 or $\infty$; and its weak dimension is 0 or $\infty$. Combining these computations with those of [7], we obtain the finitistic dimensions of $\pi_{*}$, except the injective dimension. Moreover, we obtain geometric characterizations of $\pi_{*}$-modules of projective dimensions 0,1 and weak dimension 0 . These characterizations are interesting: For projective modules they are about the cohomology groups of geometric realization; while for flat modules they are about the homology. Heuristically "projective" and "flat" are dual notions in homological algebra, while "cohomology" and "homology" are dual in topology. These characterizations show that these two heuristic

Presented to the Society, April 1, 1970 under the title Flat $\pi_{*}$-module and homological algebra of $\pi_{*}$; received by the editors November $9,1971$.

AMS (MOS) subject class ifications (1970). Primary 55E45, 55E 10.

Key words and phrases. Stable homotopy ring, stable homotopy module, finitistic dimensions, projective dimension, weak dimension, injective dimension, higher order homology operation, cohomology operation, Eilenberg-Mac Lane spectrum, Postnikov system, projective module, flat module, injective module, Puppe sequence, mapping cone sequence, spectral sequence, Hurewicz homomorphism.

(1) The preparation of this paper was sponsored by National Science Foundation Grant GP-29533. 
notions are, surprisingly, well fitted to each other. Schematically

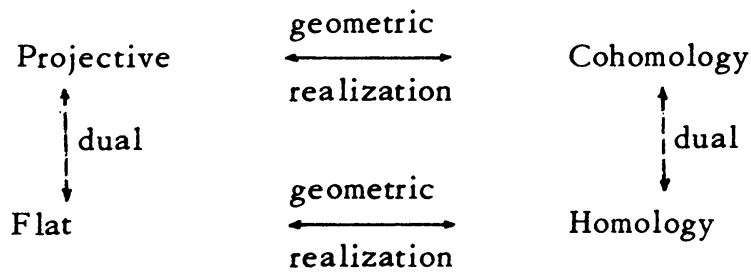

1.2 Main results. Let $M$ be, as in [7], the category of $\pi_{*}$-modules bounded from below [7, Definition 2.3]; one can, without loss of generality, regard $\mathbb{M}$ as the category of nonnegatively graded $\pi_{*}$-modules. As in [7], our spectra will be assumed to be connected, pointed CW-spectra [3]. We will not distinguish between a map and its homotopy class, nor between a spectrum and its homotopy type. A $\pi_{*}$-module which is realizable by some spectrum, say $X$, is called a stable homotopy module and is denoted by $\pi_{*}(X)$.

5.7 Theorem (Projective dimension theorem). Let $M$ be a $\pi_{*}$-module, then

(i) The projective dimension $\mathrm{P} \mathrm{d}_{\pi}, M$ of $M$ is either 0,1 or $\infty$.

(ii) $\mathrm{P} \mathrm{d}_{\pi_{*}} M=0$ iff there is a wedge $R$ of spheres such that $\pi_{*}(R) \simeq M$.

(iii) $\mathrm{P}_{\pi}^{*}, M=1$ iff there is a spectrum $L$ with trivial homology operations, which is not a wedge of spheres, such that $\pi_{*}(L) \simeq M$.

5.9 Theorem (Weak dimension theorem). Let $M$ be a $\pi_{*}$-module, then

(i) The weak dimension $\mathbb{W}_{\pi}, M$ of $M$ is either 0 or $\infty$.

(ii) $\mathbb{W} \mathrm{d}_{\pi^{*}} M=0$ iff there is a spectrum $L$ with trivial bomology operations sucb that $\pi_{*}(L) \simeq M$.

5.11 Theorem (Duality theorem). Let $M$ be a $\pi_{*}$-module, then

(i) $M$ is projective iff there is a spectrum $R$ with trivial cobomology operations such that $\pi_{*}(R) \simeq M$.

(ii) $M$ is flat iff there is a spectrum $L$ with trivial bomology operations sucb that $\pi_{*}(L) \simeq M$.

5.12 Theorem. If $M$ is a $\pi_{*}$-module, then the following conditions are equivalent.

(i) $M$ is a flat (but not free) $\pi_{*}$-module.

(ii) $\mathrm{P} \mathrm{d}_{\pi}, M=1$.

(iii) $M$ is realizable by a wedge $\bigvee_{L}\left(G_{n}, n\right)$ of Moore spectra, where each $G_{n}$ is torsion free, but at least one $G_{n}$ is not free.

(iv) $M$ is realizable by a spectrum with trivial bomology operations, which is not a wedge of spheres.

In homological algebra, for a given ring $A$, there are various dimensions-the 
so-called finitistic dimensions-attached to it ([2] or [7]). These dimensions are

$$
\begin{aligned}
\mathrm{GD}(A) & \geq \mathrm{F} \mathrm{P} \mathrm{D}(A) \geq \mathrm{fl} \mathrm{P} \mathrm{D}(A) \geq \mathrm{f} \mathrm{P} \mathrm{D}(A), \\
\mathrm{F} \mathrm{I} \mathrm{D}(A) & \geq \mathrm{FW} \mathrm{D}(A) \geq \mathrm{flW} \mathrm{D}(A) \geq \mathrm{fW} \mathrm{D}(A) .
\end{aligned}
$$

(Here we assume that $A$ is commutative in the graded sense.) For the stable homotopy ring $\pi_{*}$, we have completely determined all these dimensions, except F I D $\left(\pi_{*}\right)$. Namely,

\subsection{Theorem.}

$$
\begin{aligned}
& \mathrm{GD}\left(\pi_{*}\right)=\infty>\mathrm{FPD}\left(\pi_{*}\right)=1>\mathrm{flPD}\left(\pi_{*}\right)=\mathrm{fPD}\left(\pi_{*}\right)=0, \\
& \text { F I D }\left(\pi_{*}\right) \geq 1>\mathrm{FW} \mathrm{D}\left(\pi_{*}\right)=\mathrm{flWD}\left(\pi_{*}\right)=\mathrm{fWD}\left(\pi_{*}\right)=0 .
\end{aligned}
$$

This paper is a ring theoretical report on the stable homotopy ring of spheres, thus most of the works are algebraic. Theorems 5.7(i), 5.9(i), 5.12(i), (ii) are purely algebraic. They are essentially based only on Milnor-Kervaire's computation of Im $J$, and hold good even for a more general ring ( $\$ 4)$. One interesting "feed back" to geometry of these algebraic theorems is that they allow us to realize the flat $\pi_{*}$-modules, even the modules unbounded below (see [10]). Thus it may provide us the possibilities to study the flat modules (including those unbounded below) by geometric means. However one should note that at the present stage, we do not yet have effective means to deal with the nonconnected spectra.( $\left.{ }^{2}\right)$

The paper is organized as follows: $\$ 1$ is introduction and statement of main results. In $\$ 2$ we define the higher order homology and cohomology operation in a very general setting. In $\$ 3$ we characterize the stable spheres in terms of homology operations. In $\$ 4$ we compute the projective and weak dimensions of general $\pi_{*}$-modules. In $\oint_{5}$ we give the proofs to the main theorems stated in this section. We would suggest that the reader, after $\$ 1$, go directly to $\$ 5$ and refer to other sections when necessary.

The author would like to thank Professor William Massey for the encouragement and fruitful,discussions; he would also like to thank the referee for valuable suggestions, which shorten several proofs.

2. Higher order homology and cohomology operations. In this section we will define the higher order stable homology and cohomology operations in a very general setting. Roughly speaking, a primary homology (cohomology) operation is a natural transformation of homology functors, and a secondary operation is a natural transformation defined on the kernel of a primary operation with values in the cokernel of primary operations; by proceeding in this way, one can define the

(2) Boardman, Eilenberg and Moore are working on "spectral sequences of whole plane" which probably will be a good machinery. 
operation of any higher order. We will formulate these ideas in terms of spectral sequences, namely, the first differentials will be the primary operations and the second differentials will be the secondary operations, etc. In order to formulate these ideas more precisely we need some notations for spectral sequences.

The notion of spectral sequences that we need is slightly more general than that in standard texts. Let us recall that a bigraded abelian group $E$ is an indexed collection of abelian groups $E_{s, t}$ for every pair of integers $s$ and $t$. A differential $d: E \rightarrow E$ of bidegree $(-r, r-1)$ is a collection of homomorphisms $d: E_{s, t} \rightarrow E_{s-r, t+r-1}$, for all $s$ and $t$, such that $d^{2}=0$. The homology group $H(E)$ is the bigraded group defined by

$$
H_{s, t}(E)=\left[\operatorname{Ker}\left(d: E_{s, t} \rightarrow E_{s-r, t+r-1}\right)\right] / d\left(E_{s+r, t-r+1}\right) .
$$

2.1 Definition. A homology spectral sequence is a sequence $\left\{E^{r_{i}}, d^{r}\right\}$ such that (a) $r_{i}$ is an increasing sequence of integers. (Here is the point where we deviate from standard treatment.) (b) $E^{r_{i}}$ are bigraded abelian groups and $d^{r_{i}}$ is a differential of degree $\left(-r_{i}, r_{i}-1\right)$ on $E^{r_{i}}$. (c) There is a given isomorphism $H\left(E^{r_{i}}\right) \simeq E^{r_{i+1}}$.

Note that the differential in the homology spectral sequence is lowering the degree of the first coordinate. There are spectral sequences which raise the degree of the first coordinate. They are called cohomology spectral sequences and are defined similarly. We left the details to the reader.

A homomorphism of spectral sequences is defined as usual and their compositions are obviously still homomorphisms. Thus the collection of all spectral sequences is a category. The category of homology spectral sequences will be denoted by $\tilde{E}$.

With these notations we can now define the homology operations. Let $\Sigma$ be the category of $\mathrm{CW}$-complexes or more general $\mathrm{CW}$-spectra. For convenience we will use Boardman's CW-spectra [3]; and by his formulation one can regard $\mathrm{CW}$-complexes as some connected spectra.

2.2 Definition. Let $\mathcal{E}$ be the category of homology spectral sequences. Then a covariant functor $T: \Sigma \rightarrow \mathcal{E}$ is called a Total Homology Operation if the first term $E_{* *}^{r_{1}}$ of the spectral sequence $T(X)=\left\{E^{r_{i}}, d^{r_{i}}\right\}$ is the ordinary homology group, that is,

$$
E_{p, q}^{r_{1}}=H_{p}\left(X, G_{q}\right)
$$

where each $G_{q}$ is an abelian group. The differential $d^{r}$ is called a Primary Operation and $d^{r}$ is called a Homology Operation of the $i$ th order.

Similarly, if we take $\bar{E}$ to be the category of cohomology spectral sequence and $T$ to be a contravariant functor, then we get Total Cohomology Operation and cohomology operations of various orders. 
Example 1. Let $0 \rightarrow Z \rightarrow Z \rightarrow Z_{2} \rightarrow 0$ be an exact sequence. Then there is an exact couple<smiles>[Y4][Y4]([Y4])=O</smiles>

which gives rise to the Bockstein spectral sequence $B(X)$. Then the functor $T: \Sigma \rightarrow \mathcal{E}$ defined by $T(X)=B(X)$ is a total homology operation and its differentials are just the usual Bockstein primary and higher order operations.

Example 2. Most common operations arise from a Postnikov system. Let

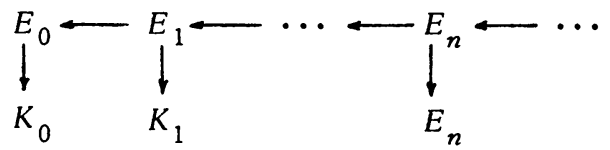

be a Postnikov system, where $K_{i}$ is an Eilenberg-Mac Lane spectrum. Then the functor $T$, which assigns to each $X \in \Sigma$ the spectral sequence of the following exact couple

$$
\begin{gathered}
\sum_{n} \pi_{*}\left(X \wedge E_{n}\right) \rightarrow \sum_{n} \pi_{*}\left(X \wedge E_{n+1}\right) \\
\sum_{n} \pi_{*}\left(X \wedge K_{n}\right)^{2}=\sum_{n} H_{*}(X),
\end{gathered}
$$

is a total homology operation, and its various differentials are various higher order homology operations.

2.3 Definition. Let $d^{r}$ be the $i$ th order homology operation of the total homology operation $T$. Then the $i$ th order homology operation $d^{r_{i}}$ is said to be trivial on spectrum $X$ if the $i$ th differential of the spectral sequence $T(X)$ is zero. The total homology operation $T$ is trivial on spectrum $X$ if all the differentials of $T(X)$ are zero. An $i$ th order homology operation $d^{r_{i}}$ is of degree $\geq n$ if $d^{r_{i}}$ sends the homology class of dimension $s$ to that of dimension $\leq s-n$. The total homology operation $T$ is of degree $\geq n$ if each $d^{r}$ is of degree $\geq n$. Similarly we can define the degree for cohomology operations.

A spectrum $X$ is called a homology (cohomology) operational trivial spectrum abbreviated as HOTS (COTS), if all the possible total homology (cohomology) operations of degree $\geq 1$ are trivial on the spectrum.

If the Postnikov system of Example 2 arises from a Postnikov decomposition of a spectrum, then the total homology operations of Example 2 are trivial on the 0 -sphere $S^{0}$ (by dimension reason). In fact $S^{0}$ is a HOTS (COTS).

Next we would like to investigate the question of when a "subspectrum" or "quotient spectrum" of a HOTS is itse lf a HOTS. We have the following proposition. 
2.4 Proposition. Let $X$ and $Y$ be spectra and suppose there are maps between $X$ and $Y$ such that, for every $G$, either

$$
0 \rightarrow H_{*}(X ; G) \rightarrow H_{*}(Y ; G)
$$

or

$$
H_{*}(Y ; G) \rightarrow H_{*}(X ; G) \rightarrow 0
$$

is exact. Then if $Y$ is a HOTS, $X$ is also a HOTS.

Proof. Let $T$ be a total homology operation; then one of the lowest order nonzero differentials on $T(X)$ (which is defined on all $H_{*}(X)$ ) certainly implies that the corresponding differentials are nonzero on $T(Y)$; a contradiction. Q.E.D.

Next we will investigate the duality between higher order homology and cohomology operations.

Let $\bar{Q}$ be the rational number modulo one. Let $\bar{G}=\operatorname{Hom}_{Z}(G, \bar{Q})$ be all the $Z$-homomorphisms of $G$ to $\bar{Q}$, where $Z$ is, as usual, the integers, Let $E=$ $\left\{E_{p, q}^{r_{i}}, d_{p, q}^{r_{i}}\right\}$ be a homology spectral sequence. Then

$$
\bar{E}=\operatorname{Hom}_{Z}(E, \bar{Q}) \equiv\left\{\bar{E}_{r_{i}}^{p, q}, \bar{d}_{r_{i}}^{p, q}\right\}
$$

defined by

$$
\bar{E}_{r_{i}}^{p, q}=\operatorname{Hom}_{Z}\left(E_{p, q}^{r_{i}}, \bar{Q}\right) \text { and } \bar{d}_{r_{i}}^{p, q}=\operatorname{Hom}_{Z}\left(d_{p, q}^{r_{i}}, \bar{Q}\right)
$$

is a cohomology spectral sequence, because $\operatorname{Hom}_{Z}(-, \bar{Q})$ is an exact functor. Let $\Sigma^{\prime}$ be the category of connected spectra with finite skeleton. Then we have

2.5 Theorem. Let $T: \Sigma^{\prime} \rightarrow \mathcal{E}$ be a total bomology (resp. cobomology) operation. Then there is a total cobomology (resp. bomology) operation $\bar{T}: \Sigma^{\prime} \rightarrow \overline{\mathcal{E}}$ satisfying the following condition: The ith order cobomology operation $\bar{d}_{r_{i}}(=$ the ith differential of $\bar{T})$ is trivial iff ith order bomology opera. tion $\bar{d}_{r_{i}}(=t$ the ith differential of $T)$ is trivial.

Proof. We will only give the proof for the homology operation $T$, since the case for cohomology can be obtained by verbatim change. Let $T(X)=\left\{E_{p, q}^{r_{i}}, d^{r^{i}}\right\}$, and define $\bar{T}$ as above. Then as remarked above $\bar{T}(X)$ is a cohomology spectral sequence. Thus $\bar{T}$ is a functor $\Sigma^{\prime} \rightarrow \overline{\mathcal{E}}$. Moreover, note that since $E_{*, *}^{r_{1}}=$ $H_{*}\left(X, G_{*}\right)$, we have

$$
E_{r_{1}}^{*, *}=\operatorname{Hom}_{Z}\left(H_{*}\left(X ; G_{*}\right), \bar{Q}\right)=H^{*}\left(X ; \bar{G}_{*}\right)
$$

where the last equality is obtained from [4, p. 120, Proposition 5.1 when $T$ is a homology operation, or Proposition 5.3 when $T$ is a cohomology operation ]. 
Thus $\bar{T}$ is a cohomology operation. Also note that it follows immediately from $\bar{d}_{r_{i}}=\operatorname{Hom}_{Z}\left(d^{r}, \bar{Q}\right)$ that $\bar{d}_{r_{i}}=0$ iff $d^{r_{i}}=0$. This completes the proof.

3. Characterization of stable spheres. In this section we will characterize the stable spheres in terms of homology or cohomology operations. From now on we will assume all the operations are of degree $\geq 1$, for example we will not regard the coefficient homomorphism as a cohomology operation.

The following theorem is more or less a folk theorem (e.g. see [8]).

3.1 Theorem. Let $X$ be a connected spectrum. Then $X$ is a wedge of spheres iff all the cohomology operations of degree $\geq 1$ on $X$ are trivial.

The dual version is slightly different, due to the incompleteness of the duality, namely,

3.2 Theorem. Let $X$ be a connected spectrum. Then $X$ is a wedge of spberes iff (i) $H_{*}(X ; Z)$ is a free abelian group, and (ii) all the bomology operations of degree $\geq 1$ on $X$ are zero.

Note that (i) is necessary; for example, the Moore spectrum of rational numbers does not admit any nonzero homology operations.

Since the proof is fairly long, perhaps it is desirable to give an outline. First we set up a lemma which establishes the fact that a map between wedges of spheres is detectable by homology operation (in general this may not be true, see [9]). Then we use the co-Postnikov system (or the homology decomposition) to show that the spectrum is obtained by attaching cells successively in a certain "normal" way. Then apply the lemma to show that all these maps are nullhomotopic.

3.3 Lemma. Let $R$ and $Y$ be wedges of spheres and $f: R \rightarrow Y$ be a map which induces a zero map $f_{*}: H_{*}(R ; G) \rightarrow H_{*}(Y ; G)$ for every $G$. Then $f$ is null-bomotopic iff the mapping cone $C_{f}$ is a HOTS.

Proof. The main idea of the proof is first, reduce the discussion of $f$ to its restriction on a finite wedge of spheres. Then, by duality, the problem is reduced to the well-known situation-detecting map by cohomology operations.

The necessity is obvious. To prove the sufficiency, let us assume, to the contrary, that $f$ is not null-homotopic. Since $R$ is a wedge of spheres, there is at least one sphere, say $S_{0}$, in $R$ such that the restriction $\phi=f \mid S_{0}$ is not nullhomotopic. Then $\phi$ determines a nonzero element, denoted by $\phi$ again, in $\pi_{*}(Y)$. Since $Y$ is a wedge of spheres (and hence $\pi_{*}(Y)$ is a free $\pi_{*}$-module), there is a finite wedge $Y^{\prime} \subset Y$ such that $\phi$ lies in $\pi_{*}\left(Y^{\prime}\right)$, which is a free direct summand of $\pi_{*}(Y)$. In other words, $\phi$ can be factored through the finite wedge $Y^{\prime}$, that is, there is $\phi^{\prime}: S_{0} \rightarrow Y^{\prime}$ such that the following diagram is commutative 


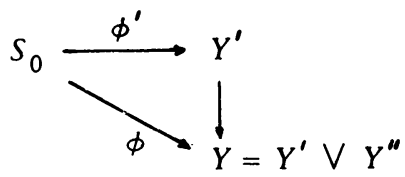

where $Y^{\prime \prime}$ is the complement of $Y^{\prime}$ in $Y$. Obviously the mapping cone $C_{\phi}=Y^{\prime} \cup_{\phi^{\prime}} C S_{0}$ is a direct summand of the mapping cone $C_{\phi}=Y \cup_{\phi} C S_{0}$, namely,

$$
C_{\phi}=C_{\phi^{\prime}} \vee Y^{\prime \prime}
$$

Next, let $R^{\prime \prime}$ be the complement of $S_{0}$ in $R$, i.e. $R=S_{0} \vee R^{\prime \prime}$. Then from the geometry we have a mapping cone sequence

$$
R^{\prime \prime} \stackrel{\psi}{\longrightarrow} C_{\phi} \stackrel{j}{\longrightarrow} C_{f}=Y \cup_{f} C R
$$

where $\psi$ is the composition $R^{\prime \prime} \subset R \stackrel{f}{\rightarrow} Y \subset C_{\phi}$. Note that $\psi$ induces a zero map $\psi_{*}: H_{*}\left(R^{\prime \prime} ; G\right) \rightarrow H_{*}\left(C_{\phi} ; G\right)$ for every $G$, since $f_{*}: H_{*}(R ; G) \rightarrow H_{*}(Y ; G)$ is, by assumption, a zero map for every $G$. Thus, from (2), we get a monomorphism

$$
0 \longrightarrow H_{*}\left(C_{\phi} ; G\right) \stackrel{\phi_{*}}{\longrightarrow} H_{*}\left(C_{f} ; G\right)
$$

for every $G$.

Now, note that from the assumption that $C_{f}$ is a HOTS, from (3) above and Proposition 2.4, we conclude that $C_{\phi}$ is a HOTS. Also note that since $C_{\phi^{\prime}}$ is a direct summand of $C_{\phi}$ (see (1) above), we can also deduce that $C_{\phi^{\prime}}$ is a HOTS. Then, by Theorem 2.5, $C_{\phi^{\prime}}$ is also a COTS (note that $C_{\phi^{\prime}}$ is finite). Thus, by Peterson's Theorem [12], $\phi^{\prime}$ is null-homotopic which is absurd. (Note that $\phi_{*}^{\prime}: H_{*}\left(S_{0} ; G\right) \rightarrow H_{*}(Y ; G)$ is zero for every $G$ because $\phi_{*}^{\prime}$ is the restriction of $f_{*}$ factoring through a direct summand $H_{*}\left(Y^{\prime} ; G\right)$ of $H_{*}(Y ; G)$; and all the cohomology operations are trivial on $C_{\phi^{\prime}}$.) Thus we conclude that $f$ is nullhomotopic.

Proof of Theorem 3.2. Since $X$ is a connected spectrum, there is a coPostnikov system (or homology decomposition) $\left\{Y_{n}\right\}$ (see [6]):

$$
\begin{aligned}
& Y_{m} \subset Y_{m+1} \subset \cdots \subset Y_{n} \subset Y_{n+1} \subset \cdots ; \quad \bigcup Y_{n}=Y \simeq X \\
& \left.\left.\prod_{m}\right|_{m} ^{\prime} k_{m+1}^{\prime} \quad \prod_{m+1} k_{n}^{\prime}\right|_{n+1} ^{\prime} \\
& R_{n} k_{n} R_{n+1}
\end{aligned}
$$

such that

(i) $H_{q}\left(Y_{n} ; Z\right)=H_{q}(X ; Z), q \leq n$; 
(ii) $H_{q}\left(Y_{n} ; Z\right)=0, q>n$;

(iii) $Y_{n+1}=C_{k_{n}^{\prime}}=Y_{n} \cup_{k_{n}^{\prime}} C R_{n}$;

(iv) $R_{n}$ is the Moore spectrum of type $\left(H_{n+1}(X), n\right)$;

(v) $k_{n^{*}}^{\prime}: H_{*}\left(R_{n} ; Z\right) \rightarrow H_{*}\left(Y_{n} ; Z\right)$ is a zero map.

Note that, by assumption, $H_{*}(X ; Z)=H_{*}(Y ; Z)$ is a free abelian group and hence each Moore spectrum $R_{n}$ is, in fact, a wedge of spheres. Thus in order to show that $Y$ is a wedge of spheres, it is sufficient to show that each $k_{n}^{\prime}$ is nullhomotopic.

We will show, by induction, that each $k_{n}^{\prime}$ is null-homotopic. In order to do this let us assume that it has been shown that each $k_{l}^{\prime}, l<n$, is null-homotopic. Then we will show that $k_{n}^{\prime}$ is null-homotopic. Note that since $k_{l}^{\prime}, l<n$, are assumed to be null-homotopic, $Y_{n}$ is a wedge of spheres. Thus $k_{n}^{\prime}: R_{n} \rightarrow Y_{n}$ is a mapping between wedges of spheres. From (v) above $k_{n^{*}}^{\prime}: H_{*}\left(R_{n} ; Z\right) \rightarrow$ $H_{*}\left(Y_{n} ; Z\right)$ is a zero map. Moreover, by the universal coefficient theorem, $k_{n^{*}}^{\prime}: H_{*}\left(R_{n} ; G\right) \rightarrow H_{*}\left(Y_{n} ; G\right)$ is zero for every group $G$ (note that both $H_{*}\left(R_{n} ; Z\right.$ ) and $H_{*}\left(Y_{n} ; Z\right)$ are free). Thus $k_{n}^{\prime}$ satisfies the conditions of Lemma 3.3 . Therefore it is sufficient to show that $Y_{n+1}$ is a HOTS. From the assumption that $H_{*}(X ; Z)$ is free, we can, by the universal coefficient theorem, improve (i) and (ii) above to the following statements: For every $G$,

(i') $H_{q}\left(Y_{n} ; G\right)=H_{q}(X ; G), q \leq n$;

(ii') $H_{q}\left(Y_{n} ; G\right)=0, q>n$.

Thus we have a monomorphism

$$
0 \rightarrow H_{*}\left(Y_{n} ; G\right) \rightarrow H_{*}(Y ; G)
$$

for every $G$. Then, by Proposition 2.4, we conclude that $Y_{n}$ is a HOTS. This proves that $k_{n}^{\prime}$ is null-homotopic. Thus, by induction, we have shown that all $k_{n}^{\prime}$ are null-homotopic and therefore $Y$ is a wedge of spheres. Q.E.D.

4. Dimension of $\pi_{*}$-modules. In this section we will compute all the possible dimensions for general $\pi_{*}$-modules.

First let us note that if $M$ is a $\pi_{*}$-module, then obviously we have $M / M \pi_{+} \simeq$ $A \otimes Z$ where $A \otimes Z$ means the tensor product over $\pi_{*}(3)$ and $\pi_{+}=\left\{\pi_{n}, n>0\right\}$ is the Jacobson radical of $\pi_{*}$.

4.1 Proposition. The rational number $Q$ is a flat $\pi_{*}$-module via augumentation.

Proof. The $Z$-flatness of $Q$ yields the following exact sequence for any $\pi_{*}$-module $M$.

$$
0 \rightarrow M \pi_{+} \otimes_{Z} Q \rightarrow M \otimes_{Z} Q \rightarrow M / M \pi_{+} \otimes Q \rightarrow 0
$$

(3) Throughout the whole paper $\otimes$ will mean tensor product over $\pi_{*}$. 
Since $M \pi_{+}$is $Z$-torsion, $M \pi_{+} \otimes_{Z} Q=0$. Therefore

$$
M \otimes_{Z} Q \simeq M / M \pi_{+} \otimes_{Z} Q \simeq(M \otimes Z) \otimes_{Z} Q=M \otimes Q .
$$

Thus $-\otimes_{Z} Q$ and $-\otimes Q$ are the same functors; and $-\otimes Q$ is an exact functor. Therefore $Q$ is a flat $\pi_{*}$-module.

4.2 Proposition. Let $M$ be a flat $\pi_{*}$-module such that $M / M \pi_{+}$is a free abelian group. Then $M$ is a free $\pi_{*}$-module.

Proof. Let $\left\{e_{i}\right\}$ be elements in $M$ such that their images $\left\{\bar{e}_{i}\right\}$ under the natural map constitute a basis of the free abelian group $M / M \pi_{+}$. Let $F$ be a free $\pi_{*}$-module with basis $\left\{x_{i}\right\}$ such that $\operatorname{deg} x_{i}=\operatorname{deg} e_{i}$. Let $f: F \rightarrow M$ be the homomorphism sending $x_{i}$ to $e_{i}$. Tensoring with $Z$, we have an isomorphism

$$
f \otimes Z: F \otimes Z \rightarrow M \otimes Z=M, M \pi_{+} \cdot
$$

From $[11$, p. 215 , Corollary 1.5$]$, we see immediately that $f$ is onto. Thus we obtain a short exact sequence

$$
0 \rightarrow \operatorname{ker} f \rightarrow F \stackrel{f}{\rightarrow} M \rightarrow 0 .
$$

By applying the functor $\operatorname{Tor}_{*, *}^{\pi *}(-, Z)$ to this short exact sequence, we have

$$
\rightarrow \operatorname{Tor}_{1}{ }^{*}(M, Z) \rightarrow(\operatorname{ker} f) \otimes Z \rightarrow F \otimes Z \rightarrow M \otimes Z \rightarrow 0
$$

where the ring of integers $Z$ is a $\pi_{*}$-module via augumentation. Since $M$ is $\pi_{*}$-flat, $\operatorname{Tor}_{1}^{\pi_{*}}(M, Z)=0$. Combining this with the isomorphism of (1), we conclude that $(\operatorname{ker} f) \otimes Z=0$. Then from [11, p. 215, Proposition 1.4] we have ker $f=0$. In other words, $M \simeq F$ is a free $\pi_{*}$-module.

4.3 Theorem. A $\pi_{*}$-module $M$ is flat if and only if its projective dimension is equal to or less than one.

Proof. Let $M$ be a flat $\pi_{*}$-module, and let $0 \rightarrow K \stackrel{j}{\rightarrow} F \rightarrow M \rightarrow 0$ be an exact sequence with $F$ being a free $\pi_{*}$-module. Then from standard homological algebra arguments (e.g. see [7, Proposition 2.4]), we see that $K$ is $\pi_{*}-f$ lat.

Consider the following commutative diagram

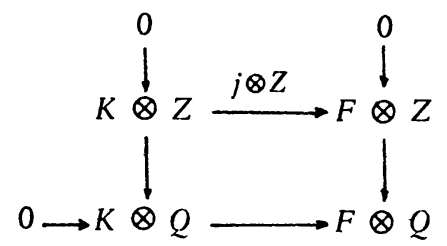

where both vertical and horizontal lines are exact. The vertical lines are exact 
because $K$ and $F$ are flat; and the bottom horizontal line is exact because $Q$ is flat (by Proposition 4.1 above). From commutativity we see easily that $j \otimes Z: K \otimes Z \rightarrow F \otimes Z$ is a monomorphism. Since $F \otimes Z$ is a free abelian group, therefore $K \otimes Z=K / K \pi_{+}$is a free abelian group too. From Proposition 4.2 , we conclude that $K$ is a free $\pi_{*}$-module. Thus the projective dimension $\mathrm{P}_{\pi} M$ of $M$ is less than or equal to 1 .

Conversely, assume that $\mathrm{P} \mathrm{d}_{\pi_{*}} M \leq 1$, then we will show that $M$ is $\pi_{*}-\mathrm{flat}$. Obviously, it is sufficient to show this for the case $\mathrm{P}_{\pi_{*}} M=1$. Let $0 \rightarrow F_{1}$ $\rightarrow F_{0} \rightarrow M \rightarrow 0$ be a free resolution of $M\left[7\right.$, Theorem 215]. Let $\left\{F_{1}^{a}, a \in J\right\}$ be a directed set of finitely generated free submodules of $F_{1}$, namely,

$$
F_{1}^{\alpha} \subset F_{1}^{\beta} \text {, if } \alpha<\beta
$$

and

$$
\bigcup_{a \in J} F_{1}^{a}=F_{1} \quad\left(=\underset{\vec{a}}{\lim } F_{1}^{a}\right)
$$

where $\lim _{\rightarrow}$ is the direct limit taken over $\left\{F_{1}^{a}, a \in J\right\}$. Obviously

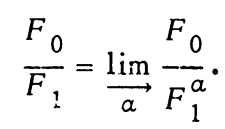

Moreover since $F_{1}^{a}$ is finitely generated, there is a finitely generated free direct summand of $F_{0}$, say $F_{0}^{\prime}$, such that $F_{1}^{a} \subset F_{0}^{\prime}$ (and $F_{0}=F_{1}^{\prime} \oplus F_{0}^{\prime \prime}$ ). Since f W D $\left(\pi_{*}\right)=0\left[7\right.$, Theorem 4.3] and $\mathrm{W} \mathrm{d}_{\pi_{*}}\left(F_{0}^{\prime} / F_{1}^{a}\right) \leq 1$, the module $F_{0}^{\prime} / F_{1}^{a}$ is flat. Hence $F_{0} / F_{1}^{a}=\left(F_{0}^{\prime} / F_{1}^{a}\right) \oplus F_{0}^{\prime \prime}$ is flat. Because the direct limit of flat modules is flat [4], we conclude that $F_{0} / F_{1}=\lim _{\rightarrow} F_{0} / F_{1}^{a}$ is flat. This completes the proof.

4.4 Theorem. The projective dimension of $\pi_{*}$-modules is either 0,1 , or $\infty$.

Proof. Assume, to the contrary, that there is a $\pi_{*}$-module $M$ of projective dimension $n>1$. Then, by a standard homological algebra argument (e.g. see $\left[7\right.$, Corollary 2.5] for similar arguments) we can assume that $\mathrm{P} \mathrm{d}_{\pi_{*}} M=2$. Then from [ 7 , Theorem 2.15], we have a free resolution of $M$,

$$
0 \rightarrow F_{2} \rightarrow F_{1} \rightarrow F_{0} \rightarrow M \rightarrow 0 .
$$

Or equivalently, we have a short exact sequence

$$
0 \rightarrow F_{1} / F_{2} \rightarrow F_{0} \rightarrow M \rightarrow 0 .
$$

Then, by Theorem 4.3, $F_{1} / F_{2}$ is a flat $\pi_{*}$-module which is embedded in a free module. By an argument similar to that in Theorem 4.3, $F_{1} / F_{0}$ is a free $\pi_{*}$-module. 
Thus $\mathrm{P} \mathrm{d}_{\pi_{*}} M=1$, a contradiction. This proves the theorem.

Remark. This theorem is true for a more general ring; for example, let $A$ be a ring satisfying (i) $A_{0} \simeq Z$ and (ii) for every prime $p$ and integer $j$ there is $a \in A_{+}$such that the order of $a$ is precisely $p^{j}$.

4.5 Theorem. The weak dimension of $a \pi_{*}$-module is either 0 or $\infty$.

Proof. Assume, to the contrary, that there is a $\pi_{*}$-module $M$ which has finite weak dimension $n \neq 0$, i.e. $\mathbb{W} \mathrm{d}_{\pi_{*}} M=n$, where $0<n<\infty$. Then from standard homological algebra (e.g. see [7, Corollary 2.5] for similar arguments), we can assume, without loss of generality, that $\mathbb{W} \mathrm{d}_{\pi_{*}} M=1$. Let us consider the following short exact sequence $0 \rightarrow K \rightarrow F \rightarrow M \rightarrow 0$ with $F$ a free $\pi_{*}$-module. Then $K$ is a flat $\pi_{*}$-module embedded in a free module (e.g. see [7, Corollary 2.4]). By arguments similar to those in Theorem 4.3, we conclude that $K$ is a free $\pi_{*}$-module. This proves that $\mathrm{Pd}_{\pi_{*}} M=1$. By Theorem 4.3, $M$ is flat, a contradiction. This completes the proof.

5. Main theorems. The present section is the central one in this paper, for here we prove the main theorems stated in $\$ 1$.

5.1 Theorem. Let $X$ be a connected spectrum, and $b: \pi_{*}(X) \rightarrow H_{*}(X ; Z)$ be the Hurewicz bomomorphism. Then $b$ is an epimorphism if all the bomology operations (of degree $\geq 1$ ) are trivial on $X$. Conversely if $H_{*}(X ; Z)$ is torsion free and $h$ is an epimorphism, then all the homology operations on $X$ are trivial.

Proof. $1^{\circ}$. Let us assume first that $X$ is a HOTS. Then we will show that the Hurewicz homomorphism $b: \pi_{*}(X) \rightarrow H_{*}(X)$ is onto. To show this, first note that there is a spectral sequence [5] $H_{*}\left(X ; \pi_{*}\right) \Rightarrow \pi_{*}(X)$ with edge homomorphism

$$
\pi_{n}(X) \rightarrow E_{n, 0}^{\infty} \subset E_{n, 0}^{2}=H_{n}\left(X ; \pi_{0}\right)=H_{n}(X),
$$

which is just the Hurewicz map. Since $X$ is a HOTS and all the differentials are homology operations of degree $\geq 1$, so $E^{2}=E^{\infty}$ whence the Hurewicz map is onto. This proves the first half of the theorem.

$2^{\circ}$. Next we will show the second half of the theorem: Let $\left\{g^{a}: s^{n a} \rightarrow X\right\}$ be a set of generators of $\pi_{*}(X)$ as a $\pi_{*}$-module, and let $M_{0}=V_{a} s^{n}$ a be the wedge of spheres $s^{n a}$. Let $f: M_{0} \rightarrow X$ be a map such that the following diagram is commutative

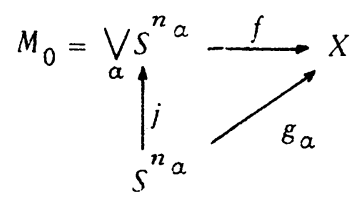

where $j$ is the natural inclusion. 
Let $\zeta_{a}$ be the fundamental class of $H_{n}\left(S^{n}{ }^{n}\right)$. Then by the definition of the Hurewicz homomorphism $b\left(g_{\alpha}\right)=g_{a *}\left(\zeta_{\alpha}\right)^{2}$ where $g_{a *}: H_{*}\left(s^{n a}\right) \rightarrow H_{*}(X)$ is induced by $g_{\alpha}$. Thus the ontoness of $b$ implies that the set $\left\{g_{a *}\left(\zeta_{\alpha}\right) \mid\right.$ for all $\left.a\right\}$ generates the whole group $H_{*}(X)$. Note that $H_{*}\left(M_{0}\right)=\Sigma_{\alpha} H_{*}\left(S^{n a}\right)$, and hence $\left\{j_{*}\left(\zeta_{\alpha}\right) \mid\right.$ for all $\left.\alpha\right\}$ generates $H_{*}\left(M_{0}\right)$. Moreover by the commutativity of the "triangle" (1) above, we have $f_{*} j_{*}\left(\zeta_{\alpha}\right)=g_{a *}\left(\zeta_{a}\right)$. Thus $f_{*} j_{*}\left(\zeta_{\alpha}\right)$ generates the whole group $H_{*}(X)$. In other words, $\operatorname{Im} f_{*}=H_{*}(X)$. That is, $f_{*}: H_{*}\left(M_{0}\right) \rightarrow H_{*}(X)$ is an epimorphism.

Note that, by assumption, $H_{*}(X ; Z)$ is torsion free; and hence, by the universal coefficient theorem, we have an epimorphism

$$
f_{*}: H_{*}\left(M_{0} ; G\right)=H_{*}\left(M_{0}\right) \otimes_{Z} G \rightarrow H_{*}(X) \otimes_{Z} G=H_{*}(X ; G)
$$

for every $G$. Now, since $M_{0}$, as a wedge of spheres, is a HOTS, we conclude from Proposition 2.4 that $X$ is a HOTS.

Thus we have completed the proof of the theorem.

5.2 Proposition. A $\pi_{*}$-module $M$ with finite projective or weak dimension is realizable as a stable bomotopy module $\pi_{*}(X)$ for some spectrum $X$, i.e. $M \simeq \pi_{*}(X)$. In particular every flat $\pi_{*}$-module is realizable.

Proof. From Theorem 4.4 , the finiteness of $\mathbb{W} \mathrm{d}_{\pi_{*}} M$ or $\mathrm{P}_{\pi_{*}} M$ implies $\mathrm{P}_{\pi_{*}} M \leq 1$. Let

$$
0 \rightarrow F_{1} \stackrel{d_{1}}{\rightarrow} F_{0} \rightarrow M \rightarrow 0
$$

be a free resolution of $M\left[7\right.$, Theorem 2.15]. Then by [7, Theorem 2.18] $F_{i}$ and $d_{1}$ can be realized by wedges of spheres $R_{i}, i=0,1$, and a map $f: R_{1} \rightarrow$ $R_{0}$. That is, $\pi_{*}\left(R_{i}\right) \simeq F_{i}$ and $f_{*}=d_{1}$. Note that the monomorphism of $d_{1}=f_{*}$ reduces the Puppe sequence of the mapping cone sequence $R_{1} \rightarrow R_{0} \rightarrow C_{f}$ into the following short exact sequence

$$
0 \rightarrow \pi_{*}\left(R_{1}\right) \rightarrow \pi_{*}\left(R_{0}\right) \rightarrow \pi_{*}\left(C_{f}\right) \rightarrow 0
$$

where $C_{f}$ is the mapping cone of $f$. Comparing the two short exact sequences above, we obtained the isomorphism $M \simeq \pi_{*}\left(C_{f}\right)$. This proves the proposition.

5.3 Theorem. $A \pi_{*}$-module $M$ is flat iff $M$ can be realized as a stable bomotopy module $\pi_{*}(X)$ by spectrum $X$ with trivial bomology operations.

Proof. It follows from Proposition 5.2 that $M$ is realizable, that is, there is a spectrum $X$ such that $M \simeq \pi_{*}(X)$. Then, from [7, Corollary 4.10], we see that $X$ is a HOTS. Thus we have shown the necessity.

To prove the sufficiency, note that from Theorem 5.1 we have an onto 
Hurewicz homomorphism $b: \pi_{*}(X) \rightarrow H_{*}(X ; Z) \rightarrow 0$. Let $M_{0}$ be the wedges of spheres which are "generators" of $\pi_{*}(X)$ as $\pi_{*}$-modules $\left(M_{0}\right.$ is constructed as in the proof $2^{\circ}$ of Theorem 5.1). Then, arguing as in the proof $2^{\circ}$ of Theorem 5.1, the ontoness of $b$ implies the ontoness of $f_{*}$.

$$
H_{*}\left(M_{0} ; Z\right) \stackrel{f_{*}}{\longrightarrow} H_{*}(X ; Z) \rightarrow 0
$$

where $f: M_{0} \rightarrow X$ is constructed as in the proof $2^{\circ}$ of Theorem 5.1.

By tensoring with abelian group $G$, we get

$$
H_{*}\left(M_{0} ; Z\right) \otimes G \rightarrow H_{*}(X ; Z) \otimes G \rightarrow 0 .
$$

Since both $X$, by assumption, and $M_{0}$ are a wedge of spheres, they are both HOTS. So the homology groups $H_{*}(X ; Z)$ and $H_{*}\left(M_{0} ; Z\right)$ are torsion free [7, Proposition 4.14]. Therefore, by the universal coefficient theorem, the above exact sequence can be rewritten as $H_{*}\left(M_{0} ; G\right) \rightarrow H_{*}(X ; G) \rightarrow 0$. Then, by the Puppe sequence of $M_{0} \stackrel{f}{\rightarrow} X \rightarrow C_{f}$, we get from the above sequence the following exact sequence $0 \rightarrow H_{*}\left(C_{f} ; G\right) \rightarrow H_{*}\left(M_{0} ; G\right)$, for every $G$. Thus, by Proposition 2.4, we conclude that $C_{f}$ is a HOTS (since $M_{0}$ is). Moreover $H_{*}\left(C_{f} ; Z\right)$, as a subgroup of a free group $H_{*}\left(M_{0} ; Z\right)$, is a free group. Therefore, by Theorem 3.2, we conclude that $C_{f}$ is a wedge of spheres. In other words, the Puppe sequence

$$
0 \rightarrow \pi_{*}\left(C_{f}\right) \rightarrow \pi_{*}\left(M_{0}\right) \rightarrow \pi_{*}(X) \rightarrow 0
$$

is a free $\pi_{*}$-resolution of $\pi_{*}(X)$. Then, by Theorem $4.3, \pi_{*}(X)$ is flat. This completes the proof.

5.4 Theorem. A spectrum with trivial bomology operations bas the same bomotopy type as a wedge of Moore spectra of tor'sion free groups.

Proof. By Theorems 5.3 and 4.3, the stable homotopy module of the HOTS $X$ has projective dimension 1 , i.e. there is a free resolution

$$
0 \rightarrow F_{1} \stackrel{d_{0}}{\longrightarrow} F_{0} \rightarrow \pi_{*}(X) \rightarrow 0 .
$$

From [ 7, Theorem 2.18], there is a realization for (1). Namely, there are wedges of spheres $R_{0}, R_{1}$ and a map $f: R_{1} \rightarrow R_{0}$ such that the following sequence

$$
0 \longrightarrow \pi_{*}\left(R_{1}\right) \stackrel{f_{*}}{\longrightarrow} \pi_{*}\left(R_{0}\right) \longrightarrow \pi_{*}(X) \longrightarrow 0
$$

$$
\begin{array}{ll}
S \| & S \| \\
F_{1} & F_{0}
\end{array}
$$


is exact and $f_{*}=d_{0}$. Note that $\pi_{*}(X)$ is flat (by Theorem 4.3), hence, by tensoring with $Z$, we still have an exact sequence

$$
0 \rightarrow F_{1} \otimes Z \rightarrow F_{0} \otimes Z \rightarrow \pi_{*}(X) \otimes Z \rightarrow 0 .
$$

From [7, Corollary 4.9] and [13], we have $\pi_{*}(X) \otimes Z=\pi_{*}(X \wedge K(Z))=H_{*}(X ; Z)$ (where $K(Z)$ is Eilenberg-Mac Lane spectrum of integers). Similarly we get $F_{1} \otimes Z=H_{*}\left(R_{1} ; Z\right)$ and $F_{0} \otimes Z=H_{*}\left(R_{0} ; Z\right)$. Let $L$ be a wedge of Moore spectra of torsion free groups such that $H_{*}(L ; Z)=H_{*}(X ; Z)$. Then it is easy to see that (3) is merely a free resolution for the group $H_{*}(L ; Z)$ which is realizable by the wedges of spheres $R_{0}, R_{1}$ and the map $f: R_{0} \rightarrow R_{1}$. Thus we have the following homotopy commutative diagram



Then, by $[3, J .12]$, there is a map $g$ such that the above diagram is homotopy commutative. Thus $g$ induces an isomorphism $H_{*}(g): H_{*}(X) \rightarrow H_{*}(L)$. By Whitehead's theorem, we have $X \simeq L$. Q.E.D.

5.5 Theorem. A $\pi_{*}$-module $P$ is projective iff $P$ can be realized as a stable bomotopy module $\pi_{*}(R)$ by spectrum $R$ with trivial cobomology operations.

Proof. The necessity follows immediately from Theorem 3.1. To prove the sufficiency, let us assume that $\pi_{*}(X)$ is projective. Then by $[1$, Eilenberg Lemma], there is a large free module $F_{1}$ such that $\pi_{*}(X) \oplus F_{1}=F_{2}$, where $F_{2}$ is a free module. Then, by [7, Theorem 2.18], we have wedges of spheres $R_{1}$ and $R_{2}$ realizing $F_{1}$ and $F_{2}$. Thus we get

$$
\pi_{*}\left(X \vee R_{1}\right) \simeq \pi_{*}\left(R_{2}\right)
$$

Moreover there is a map $f: R_{2} \rightarrow X \vee R_{1}$ which induces the above isomorphism (1); see e.g. [7, Theorem 2.18]. By Whitehead's theorem, we get $X \vee R_{1} \simeq R_{2}$. Hence we have

$$
H^{*}\left(N_{2} ; G\right)=H^{*}(X ; G) \oplus H^{*}\left(R_{1} ; G\right) .
$$

Thus $H^{*}(X ; G)$, as a direct summand of $H^{*}\left(R_{2}, G\right)$, does not admit any nonzero cohomology operations of degree $\geq 1$. Thus, by Theorem $3.1, X$ is a wedge of spheres; and hence is a HOTS. This completes the proof.

In fact we have proved more, namely,

5.6 Corollary. Every projective $\pi_{*}$-module $P$ is realizable by a wedge of spheres, and bence is free. 
5.7 Theorem. Let $M$ be a $\pi_{*}$-module. Then

(i) The projective dimension $\mathrm{P}_{\pi_{*}} M$ of $M$ is either 0,1 or $\infty$.

(ii) $\mathrm{P} \mathrm{d}_{\pi_{*}} M=0$ iff there is a wedge $R$ of spheres such that $M \simeq \pi_{*}(R)$.

(iii) $\mathrm{P}_{\pi_{*}} M=1$ iff there is spectrum $L$ with trivial homology operations, which is not a wedge of spheres, such that $M \simeq \pi_{*}(L)$.

Proof. (i) follows from Theorem 4.4; (ii) follows from Corollary 5.6; and (iii) follows from (ii) and Theorems 5.3 and 4.3 .

5.8 Corollary. $\pi_{*}(X)$ bas projective dimension $\infty$ iff there is a nonzero bomology operation on $H_{*}(X)$.

Proof. By Theorem 5.7, the projective dimension of $\pi_{*}(X)$ cannot be 0 or 1 iff $X$ is not a HOTS. So the corollary follows.

5.9 Theorem. Let $M$ be a $\pi_{*}$-module. Then

(i) The weak dimension $\mathbb{W}_{\pi_{*}} M$ of $M$ is either 0 or $\infty$.

(ii) $\mathbb{W} \mathrm{d}_{\pi_{*}} M=0$ iff there is a spectrum $L$ with trivial homology operations sucb that $M \simeq \pi_{*}(L)$.

Proof. (i) follows from Theorem 4.5, and (ii) follows from Theorem 5.3.

5.10 Corollary. W $\mathrm{d}_{\pi_{*}}\left(\pi_{*}(X)\right)=\infty$ iff there is a nonzero bomology operation on $H_{*}(X)$.

5.11 Theorem. Let $M$ be a $\pi_{*}$-module, then

(i) $M$ is projective iff there is a spectrum $R$ with trivial cobomology operations sucb that $M \simeq \pi_{*}(R)$.

(ii) $M$ is flat iff there is a spectrum $L$ with trivial bomology operations such that $M \simeq \pi_{*}(L)$.

Proof. Follows from Theorems 5.7 and 5.9.

5.12 Theorem. If $M$ is a $\pi_{*}$-module, then the following conditions are equivalent:

(1) $M$ is a flat (but not free) $\pi_{*}$-module.

(2) $\mathrm{P} \mathrm{d}_{\pi_{*}} M=1$.

(3) $M$ is realizable by a spectrum $L$ with trivial bomology operations, which is not a wedge of spheres.

(4) $M$ is a wedge $\bigvee L\left(G_{n}, n\right)$ of Moore spectra, where each $G_{n}$ is torsion free, but at least one is not free.

Proof. (1) $\Leftrightarrow$ (2) follows from Theorem 4.3. (3) $\Leftrightarrow$ (4) by Theorem 5.4. $(1) \Leftrightarrow$ (3) by Theorem 5.9. 


\subsection{Theorem.}

$$
\begin{aligned}
& \mathrm{GD}\left(\pi_{*}\right)=\infty>\mathrm{FPD}\left(\pi_{*}\right)=1>\mathrm{flPD}\left(\pi_{*}\right)=\mathrm{fPD}\left(\pi_{*}\right)=0, \\
& \text { F I D }\left(\pi_{*}\right) \geq 1>\operatorname{FWD}\left(\pi_{*}\right)=\mathrm{flWD}\left(\pi_{*}\right)=\mathrm{fWD}\left(\pi_{*}\right)=0 .
\end{aligned}
$$

Proof. fl P D $\left(\pi_{*}\right)=f P D\left(\pi_{*}\right)=f \mathbb{D}\left(\pi_{*}\right)=0$ is computed in $\left[7, \S_{1}\right.$, Theorem 3]. F P D $\left(\pi_{*}\right)=1$ and $\mathrm{FW} D\left(\pi_{*}\right)=0$ follow immediately from Theorems 4.4 and 4.5 respectively; $\mathrm{G} \mathrm{D}\left(\pi_{*}\right)=\infty$ follows immediately from the fact that F P D $\left(\pi_{*}\right) \neq \mathrm{f} \mathrm{P} \mathrm{D}\left(\pi_{*}\right)$. Thus the only thing left to be proved is that F I D $\left(\pi_{*}\right) \geq 1$.

To prove this, let us assume, to the contrary, that F I D $\left(\pi_{*}\right)=0$. Then, by $\left[2\right.$, Theorem 7.1 (c), p. 483], every $\pi_{*}$-module, in particular the integer $Z$ as $\pi_{*}$-module, contains a nonzero submodule isomorphic to a principal ideal. On the other hand one notes that every principal ideal in $\pi_{*}$ must contain some (additive) torsion element. (Let $(x)$ be a principal ideal in $\pi_{*}$ and $x \in \pi_{0}=Z$. Let $p$ be a prime which is not a factor of $x$. Then $(x)$ contains all the elements in the $p$-primary component and hence contains an (additive) torsion element.) Thus $Z$ cannot contain a submodule isomorphic to any principal ideal of $\pi_{*}$, a contradiction. This proves that F I D $\left(\pi_{*}\right) \geq 1$. Thus we have completed the proof of the theorem.

This paper has been announced in Notices Amer. Math. Soc. 17 (1970), p. 686, Abstract \#70T-G104. This author would like to apologize that he is unable to prove Theorem 5 of that article.

Open problems. (1) $\mathrm{F} \mathrm{I} \mathrm{D}\left(\pi_{*}\right)=$ ? It seems likely that $\mathrm{F} \mathrm{I} \mathrm{D}\left(\pi_{*}\right)=1$.

(2) $\operatorname{Id~}_{\pi_{*}}\left(\pi_{*}\right)=$ ? P. Freyd has shown that the self-injective dimension $\mathrm{I} \mathrm{d}_{\pi_{*}}\left(\pi_{*}\right) \neq 0$ (see Proc. La Jolla Conf. on Categorical Algebra (La Jolla, Calif., 1965), Springer-Verlag, New York, 1966, pp. 121-172). It seems to us that $\operatorname{I~d~}_{\pi_{*}}\left(\pi_{*}\right)=\infty$.

(3) $K-\operatorname{dim}\left(\pi_{*}\right)=$ ? It seems that $K-\operatorname{dim}\left(\pi_{*}\right)=1$. This question is very interesting. The Krull dimension $K-\operatorname{dim}\left(\pi_{*}\right)$ is a "measure" of prime ideals (namely, the maximal length of chains of prime ideals) and which is closely related to the nilpotency problem. For example, the Barratt's conjecture means that there is only one (nonmaximal) prime ideal in $\pi_{*}$ (which implies $K$-dim $\left(\pi_{*}\right)$ $=1$ ). Therefore the answer to this question is not only interesting by itself, it would also give a good approximation to the old question. We would like to point out here that in the theory of Noetherian ring the $K$-dim is closely related to the finitistic dimensions. If $\pi_{*}$ were a Noetherian ring (but it is not), then our computations would imply $K-\operatorname{dim} \pi_{*}=1$.

\section{REFERENCES}

1. H. Bass, Big projective modules are free. Illinois J. Math. 7 (1963), 24-31. MR $26 \# 1341$. 
2. - Finitistic dimension and homological generalization of semi-primary rings, Trans. Amer. Math. Soc. 95 (1960), 466-488. MR 28 \#1212.

4. H. Cartan and S. Eilenberg, Homological algebra, Princeton Univ. Press,

3. J. Boardman, Stable homotopy theory, University of Warwick, 1965. (mimeograph Princeton, N. J., 1956. MR 17, 1040.

5. J. M. Cohen, The decomposition of stable homotopy, Ann. of Math. (2) 87 (1968), 305-320. MR 37 \#6932.

6. P. Hilton, Homotopy theory and duality, Gordon and Breach, New York, 1965. MR $33 \# 6624$.

7. T. Y. Lin, Homological algebra of stable homotopy ring $\pi_{*}$ of spheres, Pacific J. Math. 38 (1971), 117-143.

8. - Cohomological characterization of stable spheres (mimeograph notes). 31 (1972), 617-619.

10. - Stable homotopy rings, localization and Z-graded modules, Purdue University, West Lafayette, Ind. (mimeograph notes).

11. J. W. Milnor and J. C. Moore, On the structure of Hopf algebras, Ann. of Math. (2) 81 (1965), 211-264. MR 30 \#259.

12. F. P. Peterson, Functional cohomology operations, Trans. Amer. Math. Soc. 86 (1957), 197-211. MR $21 \# 4417$.

13. G. W. Whitehead, Generalized homology theories, Trans. Amer. Math. Soc. 102 (1962), 227-283. MR $25 \# 573$.

DIVISION OF MATHEMATICAL SCIENCES, PURDUE UNIVERSITY, LAF AYETTE, INDIANA 47907 